Meta

Journal des traducteurs

Translators' Journal

\title{
Pour un vocabulaire bilingue de la production télévision
}

\section{Robert Dubuc}

Volume 26, numéro 3, septembre 1981

URI : https://id.erudit.org/iderudit/002605ar

DOI : https://doi.org/10.7202/002605ar

Aller au sommaire du numéro

Éditeur(s)

Les Presses de l'Université de Montréal

ISSN

0026-0452 (imprimé)

1492-1421 (numérique)

Découvrir la revue

Citer cet article

Dubuc, R. (1981). Pour un vocabulaire bilingue de la production télévision. Meta, 26(3), 263-268. https://doi.org/10.7202/002605ar d'utilisation que vous pouvez consulter en ligne.

https://apropos.erudit.org/fr/usagers/politique-dutilisation/ 


\title{
PROBLÈMES ET SOLUTIONS
}

\author{
POUR UN VOCABULAIRE BILINGUE DE LA PRODUCTION \\ TÉLÉVISION*
}

L'effort de francisation, entrepris il y a 20 ans à Radio-Canada, a permis d'étudier graduellement et au fur et à mesure des besoins, certaines aires du vocabulaire de la production télévision : métiers, angle de prise de vues, publicité, programmation. Toutefois ces travaux ponctuels, après vingt ans, n'avaient pas encore été intégrés à un vocabulaire bilingue global de ce domaine. Pour remédier à cette lacune, le Service de linguistique espère pouvoir souligner ses vingt années d'existence en publiant le premier vocabulaire bilingue de la production télévision. Ce vocabulaire, axé cela va sans dire sur les usages nordaméricains, cherchera à concilier - ce qui est peut-être l'inconciliable - les découpages différents de la réalité en Europe et en Amérique du Nord de manière à éviter le double écueil d'un vocabulaire d'une utilisation restreinte parce que trop exclusivement régional ou d'une terminologie trop artificielle parce non suffisamment enracinée en situation réelle.

Que cette entreprise ait pu prendre vingt ans à se matérialiser, il n'y a rien là de vraiment étonnant en raison des difficultés inhérentes à ce genre de travail : diversité des usages selon les pays, disparités des techniques mises en œuvre, faiblesse de la documentation écrite en langue française.

Très tôt cependant, nous avons eu du côté de la terminologie anglaise, d'excellents ouvrages dont la teneur terminologique était particulièrement importante. Pour ne citer que les deux principaux, mentionnons de Harry Wayne McMahan, Television Production, publié à New York chez Hastings House, en 1957, et de Rudy Bretz, Techniques of Television Production, paru en 1962, chez McGraw-Hill. Ces deux ouvrages comprennent au moins $80 \%$ du vocabulaire utilisé dans le domaine et constituent un corpus très représentatif de la terminologie spécialisée de ce secteur.

Cette terminologie est non seulement spécialisée : elle est aussi spéciale. C'est une microlangue avec son lexique et ses niveaux de langage propres. La terminologie anglaise - nord-américaine - doit comprendre environ 2500

Colloque de Glendon, 1980. 
unités terminologiques. Elle se caractérise d'abord par de nombreux emprunts aux disciplines mères : théâtre et cinéma.

$\mathrm{Au}$ théâtre, la télévision a emprunté, par exemple, la plupart de ses termes de décoration : set, practical set, flat, tormentors, wing flat; la nomenclature des rôles : character actor, lead, juvenile lead. Au cinéma, elle est allée chercher les appellations des plans de prise de vues : close-up, medium shot, knee shot, establishing shot, etc. et une bonne partie du vocabulaire des commandements de prise de vues "roll it», "change", "frame», etc. Si l'on fait un examen un peu plus attentif des origines de ce vocabulaire, il se ventile à peu près ainsi: emprunts au cinéma $30 \%$, emprunts au théâtre $11 \%$, emprunts à la publicité $7 \%$, emprunts à divers domaines: journalisme, statistique, etc. $11 \%$. Les termes nés en télévision comptent pour $41 \%$, avec bien sûr, des emprunts à la radio, en particulier en ce qui concerne l'aspect sonore de l'émission télévisuelle.

Les termes créés en télévision désignent d'abord des inventions nouvelles : videotape, videotape recording, videocassette, magnetic stripe; et aussi des adaptations à l'expression télévisuelle de techniques du théâtre ou du cinéma, dry run, run through, camera rehearsal.

Une autre caractéristique de ce vocabulaire, c'est sa richesse en expressions argotiques pour désigner des métiers : pancake turner (turntable operator), gaffer (lighting director), juicer (electrician), finder (propman specialist), gofer (errand boy). L'argot apparaît aussi pour désigner certains appareils : cans (head set), crawl (title drum), cookie (gobo). La proportion des termes argotiques dans l'ensemble du vocabulaire de la production reste très difficile à évaluer à cause de sa grande volatilité. Les mots naissent ainsi à profusion, mais souvent ces créations sont éphémères ou restent d'un usage très circonscrit.

Par contre, l'usage des abréviations en langue parlée offre dans ce secteur des caractéristiques particulières. Peut-être est-ce à cause de l'usage abondant des formes abrégées utilisées par commodité dans les découpages, ou par besoin de faire court à tout prix, toujours est-il que beaucoup d'expressions sont couramment désignées par leurs abréviations dans les studios; mentionnons à titre d'exemples: T.D. pour Technical Director, T.P. pour test pattern, V.O pour Video Operator, A. D pour Art Director.

Dans diverses techniques, le vocabulaire anglais utilisé au Canada est souvent déchiré entre deux pôles d'attraction divergents : les États-Unis et la Grande-Bretagne. En télévision, la puissance de l'industrie américaine a vite fait basculer la balance en sa faveur, en dépit du fait que la B.B.C. a pour ainsi dire, tenu Radio-Canada sur les fonts baptismaux. Le seul vestige de cette parenté spirituelle reste peut-être l'emploi du mot producer, conformément à l'usage britannique, pour désigner le maître d'œuvre d'une émission, contrairement à l'usage américain qui emploie le mot director en ce sens. On peut donc dire sans exagération que le vocabulaire de la télévision canadienne anglaise est essentiellement tributaire de la terminologie américaine.

Voilà donc, brossé à grands traits, le portrait du vocabulaire anglais de la production télévision. Risquons maintenant un œil du côté du vocabulaire français correspondant. 
La terminologie française de la télévision au Canada s'est mise au point assez laborieusement. Il y a d'abord eu invasion massive de calques et d'emprunts rapportés par techniciens et réalisateurs formés à New York : continuité (au sens de scénario), tape (bande), taping (démarquage), VTR (magnétoscopie). Quant à la langue des commandements en studio, les emprunts intégraux y fleurissent: cut to, switch, take one, take two. C'est donc à l'enseigne du mélange des langues que la production télévision a fait ses premiers balbutiements terminologiques.

Mais les usagers avaient mauvaise conscience à l'égard de ce qu'ils appelaient l'abâtardissement de leur langue technique. Aussi, lorsqu'en 1960 se fonde le Comité de linguistique, les demandes affluent pour «franciser» la terminologie de la langue des studios.

Pour répondre à ces demandes, les terminologues sont allés puiser dans le vocabulaire français du cinéma, en particulier chez Jean Giraud, dont l'ouvrage constitue le premier travail terminologique consacré au cinéma. Ainsi la télévision a pu intégrer la nomenclature française des angles de prises de vue : très gros plan, gros plan, premier plan, plan moyen, plan américain, plan d'ensemble, de même que celle des mouvements de caméra: panoramique vertical, horizontal, travelling avant, travelling arrière, plongée, contre-plongée, contre-champ, etc. Au cinéma, nous avons aussi emprunté la «ponctuation» du discours en image : fondus, fondu enchaîné, volets et rideaux.

Lorsque la télé américaine a fait des emprunts au théâtre, nous avons fait de même, en particulier pour la décoration et certaines installations comme les cintres, le jeu d'orgues, etc. La radio de son côté nous léguait le vocabulaire de l'audio.

Vers 1965, certaines revues spécialisées ont commencé à paraître en France, ce qui nous a permis, grâce à un travail suivi de dépouillement, de rapprocher le vocabulaire en usage ici de celui utilisé en France. C'est ainsi que la GRILLE a remplacé l'horaire, la STATION le poste, le CRÉNEAU le time slot, l'INTERLUDE, l'intermède, etc.

Il restait pourtant des aires de différenciation difficilement conciliables qui tenaient d'une part aux emprunts ou pseudo-emprunts à l'anglais et d'autre part à la disparité des structures.

$\mathrm{Au}$ chapitre des emprunts, SPEAKER et SPEAKERINE en usage en France n'ont pas passé ici. ANNONCEUR, implanté dès les premières heures de la radio, a gardé ses positions, même si le féminin lui faisait défaut. Les noms de métiers en -man ont également suscité, à l'exception de caméraman, une réaction de rejet: personne n'a jamais voulu employer perchman, trucman, cableman, travellingman en dépit de leur usage fréquent dans les studios des radiotélévisions francophones.

D'autres écarts sont attribuables à la disparité des structures. L'émission de télévision n'est pas organisée en France comme elle l'est ici. Nous n'avons pas de directeur de la photographie, ni d'ouvriers de plateau, ni de perruquiers. Les fonctions qu'ils remplissent sont assumées par d'autres métiers. Le mot PRODUCTEUR désigne à la télévision française celui qui conçoit et réalise son émission. Cette notion n'existe pas dans nos organigrammes. Les Français ont 
des «ingénieurs du son"; plus modestes - et pour ne pas déplaire à l'Ordre des ingénieurs - nous avons des techniciens du son.

Si l'on compare maintenant le vocabulaire français de la télévision au vocabulaire américain, on constate d'abord qu'il est moins volumineux. Avec un millier de termes, la télévision d'expression française arrive à traduire ses réalités. La synonymie y est moins riche, l'argot beaucoup moins présent et l'utilisation des abréviations dans la langue parlée à peu près nulle.

Ces différences auront donc des répercussions sur la méthodologie à suivre pour l'établissement d'une terminologie comparée. Pour structurer la recherche et éviter qu'elle ne se perde dans le labyrinthe d'une terminologie encore mal assise, il convenait de trouver des pôles de regroupement de l'information terminologique; en somme, diviser en sous-domaines, le domaine général de la production télévision. Il serait ainsi plus facile de cataloguer les notions et d'établir avec à-propos les équivalences ou les correspondances interlangues. Ces six sous-domaines comprennent d'abord :

- la décoration (éléments de décors, éclairage, conception, maquillage et costumes)

- l'équipement (matériel technique de production, caméra, microphone, consoles de l'image et du son, télécinéma, magnétoscopie)

- l'installation (studios, régies, réseaux et chaînes, salles de répétition)

- la mise en scène (plans, mouvements de caméra, jeu des comédiens, textes, scénario, tournage)

- la gestion (budget, contrats, droits d'auteurs et redevances, engagements)

- la programmation (politiques, grilles, genres d'émissions, publicité, indicatifs, information)

Cette structure en place, il s'agit maintenant de faire le recoupement des notions. Cette tâche sera facilitée par le travail d'un Philippe Desjardins, ex-chef de la traduction au Siège social, qui s'est livré pendant près de dix ans au dépouillement systématique des revues de vulgarisation française, à l'affût de la terminologie d'usage. Son travail sera d'un apport inestimable pour dérégionaliser les usages locaux: par exemple détrôner enregistreuse à ruban au profit de magnétophone, ruban pour bande, passer sur les ondes pour passer à l'antenne, etc.

Dépouiller n'est cependant qu'une partie du travail. Il faut ensuite se livrer à l'analyse notionnelle du matériel recueilli. L'analyse des notions se révèle particulièrement impérieuse quand il s'agit d'un terme polysémique comme $B R O A D$ CASTER.

$\mathrm{Au}$ sens générique, ce terme désigne tous ceux qui de près ou de loin, à quelque titre que ce soit, sont mêlés à la radiotélévision. Il se rendra alors par GENS DE RADIOTÉLÉVISION, sur le modèle de gens de lettres.

Le mot broadcaster peut encore désigner les créateurs qui œuvrent en radiotélévision. Ici nous avons les TÉLÉASTES, mot créé sur le modèle de cinéaste et qui a engendré le générique RADIOTÉLÉASTE.

Le mot s'applique encore à la personne qui, professionnellement, fait des communications de nature journalistique, scientifique ou artistique, à la radio ou à la télévision. Cette notion se rendra par le néologisme COMMUNICATEUR. 
Enfin le terme désigne ceux qui exploitent des stations de radio et de télévision. Deux solutions s'offrent en français: DIFFUSEUR pour désigner la personne physique et RADIOTÉLÉVISION pour désigner l'entreprise ou la personne morale.

Ce mot de RADIOTÉLÉVISION appelle lui aussi une analyse assez serrée du contenu notionnel en raison de sa polysémie.

Le mot désigne d'abord une technique de diffusion d'un message par ondes radioélectriques. Il correspond alors en anglais à broadcasting .

Il s'applique aussi à l'ensemble des installations (stations et réseaux) dont disposent un pays ou une région pour diffuser des messages par ondes radioélectriques. Il se rend alors en anglais par broadcasting system.

Le mot désigne en outre l'industrie qui permet cette diffusion; en anglais broadcasting industry.

Enfin le mot peut s'appliquer à un organisme qui exploite des stations et des réseaux. Il correspond alors à broadcaster.

Comme on le voit, rien n'est aussi simple qu'il peut le paraitre de prime abord. Ce qui peut sauver un ouvrage de terminologie comparée de l'erreur, voire de la sottise, c'est l'analyse rigoureuse des notions. C'est à partir de cette analyse qu'il deviendra possible de recouper les notions et d'établir les équivalences qui s'imposent.

C'est aussi cette analyse qui permettra de démêler l'écheveau des synonymes, de circonscrire leur interchangeabilité, soit d'après leur sens, soit d'après leurs aires d'utilisation.

Ainsi turntable operator et pancake turner sont des synonymes, mais le dernier est de niveau argotique et ne peut s'employer que dans la langue parlée en studio. Les termes ne sont donc pas interchangeables et l'équivalent français OPÉRATEUR-DISQUES ne peut valoir strictement que pour le premier de ces termes.

Ainsi l'étude du vocabulaire de la production télévision aura comporté trois étapes: dépouillement de la documentation, analyse des notions et recoupements des notions d'après l'identification des traits sémantiques pertinents. Cette méthode garantit à notre avis la justesse des renseignements fournis et la valeur de la correspondance des termes en usage dans les deux langues.

C'est l'objectif que doit atteindre un travail de terminologie qui se veut sérieux.

ROBERT DUBUC

\section{BIBLIOGRAPHIE}

BACHY, Françoise (1971): Terminologie de la télévision, (thèse), Louvain (Belgique, Université catholique, département de Communication sociale, (ronéotypé), pagination continue.

BESSY, Maurice, Jean-Louis CHARDANS (1965) : Dictionnaire du cinéma et de la télévision, Paris, Jean-Jacques Pauvert Éditeur, 4 vol. pagination discontinue.

BRETZ, Rudy (1962): Techniques of Television Production, New York, McGraw-Hill Book Company, $2^{\mathrm{e}}$ éd., $517 \mathrm{p}$. 
Comité de linguistique $(1960+)$ : Fiches et Bulletin C'est-à-dire, Montréal, Société RadioCanada, (sans périodicité fixe).

Conseil international de la langue française (1972): Vocabulaire de la radiodiffusion, Paris, Hachette-CILF, 100 p.

Conseil international de la langue française (1972): Enregistrement restitution des images et des sons, Paris, CILF, non paginé.

DEBBASCH, Charles (1967): Traité du Droit de la radiodiffusion (radio et télévision), Paris, Librairie générale de droit et de jurisprudence, $608 \mathrm{p}$.

DUBUC, Robert (1979) : Vocabulaire bilingue du théâtre, Montréal, Leméac-Radio-Canada $174 \mathrm{p}$.

GIRAUD, Jean (1958): Le lexique français du cinéma des origines à 1930. Paris, Centre national de la recherche scientifique, $263 \mathrm{p}$.

McMAHAN, H.W. (1957): Television Production, New York, Hasting House Publishers, $231 \mathrm{p}$.

Service éditions-publications de l'ORTF (1973): ORTF 73, Paris, Presses de la cité, 701 p.

VERPRAET, Georges (1972): La Télévision et ses métiers, Paris, Bordas, coll. "BordasConnaissances", $280 \mathrm{p}$. 